\title{
Serum adipocyte-fatty acid binding protein level is elevated in severe OSA and correlates with insulin resistance
}

\author{
D.C-L. Lam*, A. Xu**, K.S-L. Lam**\#, B. Lam*, J.C-M. Lam*, \\ M.M-S. Lui* and M.S-M. Ip*,
}

ABSTRACT: Obstructive sleep apnoea (OSA) is associated with insulin resistance and metabolic syndrome. There is evidence that adipocyte-fatty acid binding protein (A-FABP) may be involved in the development of cardiometabolic dysfunction. The present authors hypothesise that A-FABP is upregulated in OSA.

A total of 124 males without hypertension, diabetes mellitus, hyperlipidaemia or cardiovascular disease were recruited and underwent polysomnography. Serum A-FABP levels showed significant positive correlations with duration of oxygen desaturation and minimal oxygen saturation, fasting insulin and insulin resistance index by homeostasis model assessment. When subjects were divided into tertiles according to apnoea/hypopnoea index (AHI), serum A-FABP levels were significantly higher in the group with $\mathrm{AHI} \geqslant 34.4$ events $\cdot h^{-1}$ than the groups with $\mathrm{AHI}$ 13.2-34.4 events $\cdot h^{-1}$ or with AHI $<13.2$ events $\cdot h^{-1}$. Serum A-FABP levels were significantly higher in the $\mathrm{AHI} \geqslant 34.4$ group than obesity-matched subjects with $\mathrm{AHI}<\mathbf{3 4 . 4}$ events $\cdot \mathrm{h}^{-1}$.

Serum adipocyte-fatty acid binding protein levels correlated with obstructive sleep apnoea and insulin resistance, independently of obesity, and were significantly higher in severe obstructive sleep apnoea. Adipocyte-fatty acid binding protein may play a role in obstructive sleep apnoea and metabolic dysfunction.

\section{KEYWORDS: Adipocyte-fatty acid binding protein, obstructive sleep apnoea}

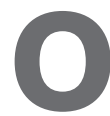
bstructive sleep apnoea (OSA) is highly associated with obesity and other components of the metabolic syndrome [1-3]. This clustering of OSA with cardiometabolic risk factors may imply that there is a common molecular pathway or mechanism underlying these disorders which has not been completely worked out. Visceral fat acts as a reservoir for various mediators, which are released into the circulation, and may play pathogenetic roles in metabolic or vascular dysfunction; OSA may act as a trigger to initiate or maintain the expression and release of these mediators [4-6].

Fatty acid binding proteins (FABPs) are a family of small intracellular lipid-binding proteins that reversibly bind hydrophobic ligands, which influence energy metabolism and inflammation, such as long chain fatty acids and eicosanoids [7]. Adipocyte-FABP (A-FABP), also known as aP2 or FABP4, is a cytosolic protein present in an abundant amount in adipocytes and macrophages [8]. A growing body of evidence from both animal and clinical studies suggests that A-FABP plays an important role in the interaction between lipid metabolism and inflammation, and may be involved in the development of the metabolic syndrome $[9,10]$. The pro-inflammatory role of A-FABP expression is supported by the observation that its expression in macrophages is induced by oxidised low-density lipoprotein (LDL) [11], as well as toll-like receptor activators [8]. In animal A-FABP and mal1 (a minor form of FABP in adipose tissue) were protected from the development of insulin resistance in the context of dietinduced obesity [12], type 2 diabetes [13], fatty liver disease [14] and hypercholesterolaemia atherosclerosis [10]. Furthermore, obese mice (ob/ob) lacking A-FABP and mal1 (aP2/mal1-/-) were able to maintain normal blood glucose levels and peripheral insulin sensitivity despite being severely obese, implying that the A-FABP deficiency genotype is associated with improved systemic insulin sensitivity, which is independent of leptin action [15]. A recent report on BMS309403, a potent inhibitor of A-FABP, showed that treatment of obese mice $(o b / o b)$ with this selective inhibitor led experiments, it has been shown that mice lacking

\section{AFFILIATIONS}

*Dept of Medicine, and

${ }^{\#}$ Research Center of Heart, Brain, Hormone and Healthy Aging,

University of Hong Kong, Hong Kong.

\section{CORRESPONDENCE}

M.S-M. Ip

University Dept of Medicine

Queen Mary Hospital

University of Hong Kong

102

Pokfulam Road

Hong Kong

Fax: 85228162863

E-mail: msmip@hkucc.hku.hk

Received:

May 162008

Accepted after revision:

October 032008

SUPPORT STATEMENT

This study was jointly supported by the Research Grants Council (RGC)

Earmarked Competitive Grant award 7667/07M, the RGC Central Allocation Grant (HKU 2/07C) and the Lee Wing Tat Cardiorespiratory Research Fund

\section{STATEMENT OF INTEREST}

Statements of interest for D.C-L. Lam, J.C-M. Lam and M.S-M. Ip can be found at www.erj.ersjournals.com/ misc/statements.shtml 
to reduced hyperglycaemia and hyperinsulinaemia [16]. A-FABP has also been shown to be involved in the mediation of allergic airway inflammation, implicating its role in asthma and possible action in bronchial epithelial cells [17].

Although traditionally thought to be a cytoplasmic protein, AFABP is also detectable in the human bloodstream. Recent evidence demonstrates circulating A-FABP level to be an independent predictor of the development of metabolic syndrome and diabetes after adjustment for the effects of adiposity $[18,19]$. In addition, serum A-FABP level has been shown to be closely associated with carotid atherosclerosis in Chinese females [20]. These epidemiological observations were also corroborated by a recent large population-based genetic study showing a reduced risk for hypertriglyceridaemia, type 2 diabetes and coronary artery disease in subjects carrying a functional genetic variant of the A-FABP gene that resulted in reduced adipose tissue A-FABP gene expression [21].

In light of the close association between OSA, adiposity and insulin resistance, the aim of the present study was to evaluate the role of A-FABP as a potential mediator in the development of metabolic dysfunction in OSA. The present authors hypothesise that severe OSA may lead to upregulation of AFABP production, which correlates with insulin resistance, promoting the development of cardiometabolic dysfunction.

\section{METHODS AND MATERIALS}

\section{Subject characteristics}

Consecutive male subjects (aged $\geqslant 18$ yrs) undergoing overnight polysomnography (PSG) at the Sleep Laboratory of Queen Mary Hospital (Hong Kong) were recruited from the period commencing October 2002 until March 2006 (excluding the period March-September 2003 when sleep laboratory services were suspended) in order to explore various cardiometabolic aspects in relation to OSA; subjects without other prevailing comorbidities were targeted (Obstructive Sleep Apnoea Cardio-Metabolic Study (OSACMS) cohort). The present authors investigated the relationship between serum A-FABP levels and sleep disordered breathing. The exclusion criteria were as follows: known history of hypertension or diabetes mellitus (fasting plasma glucose $\geqslant 7.0 \mathrm{mmol} \cdot \mathrm{L}^{-1}$ ); hyperlipidaemia on lipid-lowering therapy; cardiovascular diseases or other major chronic medical illness; use of regular medication.

All subjects gave written informed consent. The study protocol was approved by the Hong Kong University/Hong Kong Hospital Authority (Hong Kong) Institutional Review Board Ethics Committee.

\section{Anthropometric and biochemical measurements}

Clinical and anthropometric measurements (body height, body weight, waist circumference, body mass index (BMI)) were collected as previously reported [2]. Plasma glucose was measured using the glucose oxidase method with a Beckman Autoanalyzer (Beckman Instruments, Bream, CA, USA). Serum insulin was determined with a microparticle enzyme immunoassay on the Abbott IMx system (Abbott, Abbott Park, IL, USA). The insulin resistance index estimated by the homeostasis model assessment method (Homeostasis Model
Assessment - Insulin Resistance (HOMA-IR)) was calculated as follows [22]:

$$
\begin{gathered}
\text { fasting serum insulin }\left(\mu \mathrm{U} \cdot \mathrm{mL}^{-1}\right) \times \text { fasting plasma } \\
\text { glucose }\left(\mathrm{mmol} \cdot \mathrm{L}^{-1}\right) / 22.5
\end{gathered}
$$

Plasma total cholesterol and triglyceride were measured enzymatically using a Hitachi 912 Analyzer (Roche Diagnostics, $\mathrm{GmbH}$, Mannheim, Germany). High-density lipoprotein cholesterol was measured using a homogenous method with polyethylene glycol-modified enzymes and alpha-cyclodextrin. LDL cholesterol was calculated using the Friedewald equation.

\section{PSG}

PSGs were performed using a computerised diagnostic system (Alice 3 or 5; Respironics, Pittsburgh, PA, USA) in the Sleep Laboratory [2]. Manual scoring of sleep and respiratory events was performed according to standard criteria [23, 24].

\section{ELISA for quantitative measurement of human A-FABP}

Serum A-FABP levels were measured using a human A-FABP ELISA kit (Biovendor Laboratory Medicine Inc., Modrice, Czech Republic). Serum samples were diluted 1:10 before assay, then $100 \mu \mathrm{L}$ of diluted sera, calibrators and qualitycontrol samples were applied to 96-well microtitre plates coated with an affinity-purified goat anti-human FABP antibody. A calibration curve was constructed by plotting the absorbance values at $450 \mathrm{~nm}$ versus the A-FABP concentrations of the calibrators, and concentrations of unknown samples were found using this calibration curve. The intra- and interassay variations were $3.7 / 6.4 \%$ and $2.6 / 5.3 \%$ for high/low quality controls, respectively [25].

\section{Data analysis}

Sleep parameters, including apnoea/hypopnoea index (AHI), duration of desaturation, arousal index and minimum oxygen saturation, were log or logit-transformed as appropriate for further statistical analysis. Pearson correlation coefficients and partial correlation coefficients with adjustment for age, waist and BMI, were used to examine the association between serum A-FABP levels with various anthropometric, biochemical and sleep parameters. Comparisons of group means were performed using unpaired t-tests, with assumption of unequal variance (for comparison between any two groups) and using one-way ANOVA (for multiple group comparison) where appropriate. Matrix contrast examination was performed where significant variation in univariate ANOVA was seen, to examine for between-group effects. All data are expressed as mean \pm SD and $p$-values $<0.05$ were considered to be statistically significant.

\section{RESULTS}

A total of 128 males, recruited between October 2002 and March 2006, were analysed. Four subjects were excluded due to either extreme hyperlipidaemia (total cholesterol $9.0 \mathrm{mmol} \cdot \mathrm{L}^{-1}$ and triglyceride $25.6 \mathrm{mmol} \cdot \mathrm{L}^{-1}$ ) or insufficient total sleep time $(<240$ mins) at PSG. The demographic characteristics of the subjects are summarised in table 1 . Of the subjects, $96(77.4 \%)$ had OSA with an AHI $\geqslant 5$ events $\cdot h^{-1}$.

Serum A-FABP levels showed significant correlations with waist circumference and BMI (table 2). On controlling for the effects of age and obesity (BMI and waist circumference), 
TABLE 1 Clinical characteristics, sleep parameters and adipocyte-fatty acid binding protein (A-FABP) of the recruited patients and between-group comparisons of the tertile groups

\begin{tabular}{|c|c|c|c|c|c|}
\hline & \multirow[t]{2}{*}{ Overall } & \multicolumn{3}{|c|}{ AHI events $\cdot h^{-1}$} & \multirow[t]{2}{*}{ p-values $\#$} \\
\hline & & $<13.2$ & $13.2-34.4$ & $\geqslant 34.4$ & \\
\hline Subjects $n$ & 124 & 41 & 42 & 41 & \\
\hline Age yrs & $44.5 \pm 9.7$ & $40.2 \pm 8.2$ & $47.4 \pm 9.3$ & $45.9 \pm 10.2$ & 0.001 \\
\hline Waist circumference $\mathrm{cm}$ & $93.8 \pm 9.5$ & $92.5 \pm 9.0$ & $91.9 \pm 9.2$ & $97.2 \pm 9.5$ & 0.021 \\
\hline Current/ex-alcohol drinkers $\mathrm{n}$ & 64 & 20 & 25 & 19 & 0.440 \\
\hline AHI ${ }^{\bullet}$ events $\cdot h^{-1}$ & $27.6 \pm 24.3$ & $4.3 \pm 3.7$ & $20.4 \pm 5.7$ & $58.2 \pm 13.9$ & $<0.001^{\S}$ \\
\hline Duration of $\mathrm{Sa}, \mathrm{O}_{2}<\mathbf{9 0 \%}$ mins & $45.7 \pm 69.3$ & $3.2 \pm 4.2$ & $20.8 \pm 23.0$ & $113.6 \pm 83.6$ & $<0.001^{\mathrm{s}}$ \\
\hline Arousal index & $30.5 \pm 20.0$ & $14.4 \pm 6.9$ & $26.9 \pm 9.3$ & $50.3 \pm 20.4$ & $<0.001^{\S}$ \\
\hline Minimum $\mathrm{Sa}, \mathrm{O}_{2} \%^{+}$ & $74.4 \pm 14.0$ & $84.7 \pm 7.4$ & $76.8 \pm 8.6$ & $62.0 \pm 14.2$ & $<0.001^{\S}$ \\
\hline A-FABP level $n g \cdot \mathrm{mL}^{-1}$ & $16.6 \pm 6.9$ & $15.6 \pm 5.6$ & $15.1 \pm 5.4$ & $19.3 \pm 8.7$ & $0.030^{5}$ \\
\hline
\end{tabular}

Data are presented in mean $\pm \mathrm{SD}$, unless otherwise stated. AHI: apnoea/hypopnoea index; BMI: body mass index; Sa, $\mathrm{O}_{2}$ : oxygen saturation. ${ }^{\#}$ : between-group comparisons were performed using ANOVA; ${ }^{\bullet}$ : log-transformed before analysis, ${ }^{+}$: logit transformed before analysis. ${ }^{\S}$ : significance taken at $p<0.05$ for between-group comparisons after adjustment for age, waist and BMI.

serum A-FABP levels still demonstrated significant correlations with the duration (mins) of oxygen desaturation $\left(\mathrm{Sa}_{\mathrm{a}}, \mathrm{O}_{2}\right)$ $<90 \%$ during sleep $(\mathrm{r}=0.293, \mathrm{p}=0.001)$ and the minimum $\mathrm{Sa}_{\mathrm{a}} \mathrm{O}_{2}$ $(r=-0.255, p=0.004)$. Serum A-FABP also had significant correlations with fasting serum insulin levels $(r=0.343$, $\mathrm{p}<0.001)$ and HOMA-IR $(\mathrm{r}=0.333, \mathrm{p}<0.001$; table 2$)$. There was no significant correlation between serum A-FABP levels and serum lipid levels, with the exception of serum triglycerides $(r=0.284, p=0.001$; table 2$)$.
The whole group of study subjects was divided into three groups according to their AHI in tertiles, as follows. AHI Group 1: <13.2 events $\cdot \mathrm{h}^{-1}$; AHI Group 2: $13.2-34.4$ events $\cdot \mathrm{h}^{-1}$; AHI Group 3: $\geqslant 34.4$ events $\cdot h^{-1}$ (table 1 ). After adjustment for age, waist and BMI, between-group comparisons showed significant differences in AHI $(\mathrm{p}<0.001)$, duration of $\mathrm{Sa}_{\mathrm{a}} \mathrm{O}_{2}$ $<90 \%(\mathrm{p}<0.001)$, arousal index $(\mathrm{p}<0.001)$, minimum $\mathrm{Sa}_{\mathrm{O}} \mathrm{O}_{2}$ level $(\mathrm{p}<0.001)$ and serum A-FABP levels $(\mathrm{p}=0.030$; table 1$)$. Contrast examination ( $\mathrm{K}$ matrix) to test for between-group

TABLE 2 Correlations of adipocyte-fatty acid binding protein (A-FABP) with studied parameters, adjusted for waist and body mass index (BMI)

\begin{tabular}{|c|c|c|c|c|}
\hline & \multicolumn{4}{|c|}{ A-FABP $\mathrm{ng} \cdot \mathrm{mL}^{-1}$} \\
\hline & r-values & $p$-values & r-values & $p$-values \\
\hline Age yrs & -0.104 & 0.252 & & \\
\hline Waist circumference $\mathrm{cm}$ & $0.304^{\S}$ & $0.001^{\S}$ & & \\
\hline Duration of $\mathrm{Sa}, \mathrm{O}_{2}<90 \%$ mins & $0.293^{\S}$ & $0.001^{\S}$ & 0.254 & $0.005^{\S}$ \\
\hline Arousal index & 0.104 & 0.248 & 0.157 & 0.085 \\
\hline Minimum $\mathrm{Sa}_{1} \mathrm{O}_{2} \%^{+}$ & $-0.255^{\S}$ & $0.004^{\S}$ & -0.230 & $0.012^{\S}$ \\
\hline Cholesterol mmol. $\mathrm{L}^{-1}$ & 0.146 & 0.105 & 0.131 & 0.152 \\
\hline Triglyceride ${ }^{\top} \mathrm{mmol} \cdot \mathrm{L}^{-1}$ & $0.284^{\S}$ & $0.001^{\S}$ & 0.241 & $0.008^{\S}$ \\
\hline LDL mmol. $\mathrm{L}^{-1}$ & 0.013 & 0.889 & -0.027 & 0.765 \\
\hline HOMA-IR & $0.333^{\S}$ & $<0.001^{\S}$ & 0.207 & $0.022^{\S}$ \\
\hline
\end{tabular}

AHI: apnoea/hypopnoea index; $\mathrm{Sa}_{1} \mathrm{O}_{2}$ : oxygen saturation; LDL: low-density lipoprotein; HDL: high-density lipoprotein; HOMA-IR: Homeostasis Model Assessment - Insulin

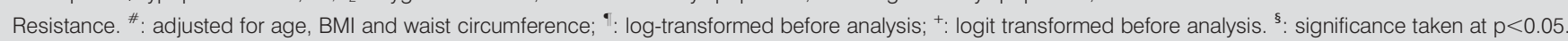




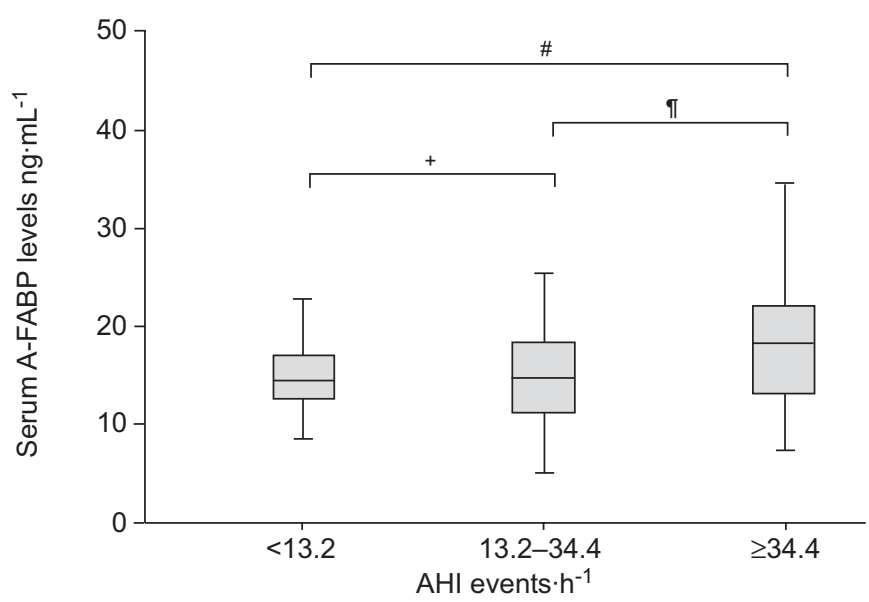

FIGURE 1. Significant difference in serum adipocyte-fatty acid binding protein (A-FABP) levels between tertile groups divided according to apnoea/hypopnoea index $(\mathrm{AHI})$. Boxes show the median and the whiskers show the range. ${ }^{*}: p=0.018$ $\because: p=0.026 ;{ }^{+}: p=0.812$.

differences showed that there were significant differences in serum A-FABP levels between AHI Group 3 and Group 1 $(p=0.018)$, and between AHI Group 3 and Group $2(p=0.026)$, but not between AHI Group 1 and Group 2 ( $p=0.812$; fig. 1).

Based on the difference between AHI Group 3 and the other AHI groups, an AHI of 34.4 events $\cdot h^{-1}$ was chosen as a cut-off level for further comparison. When comparing AHI Group 3 subjects $(n=40)$ with waist-matched subjects with an AHI $<34.4$ events $\cdot h^{-1}(n=40)$, serum A-FABP levels in AHI Group 3 subjects were significantly higher $(\mathrm{p}=0.036$; table 3$)$.

\section{DISCUSSION}

The present study demonstrates that serum A-FABP levels were significantly raised in otherwise healthy males with severe OSA compared with subjects with no or less severe OSA. A-FABP levels also showed significant correlations with sleep hypoxaemia parameters, including duration of oxygen

\begin{tabular}{|c|c|c|c|c|}
\hline \multirow[t]{3}{*}{ TABLE 3} & \multicolumn{4}{|c|}{$\begin{array}{l}\text { Serum adipocyte-fatty acid binding protein (A- } \\
\text { FABP) level in subjects with severe obstructive } \\
\text { sleep apnoea (apnoea/hypopnoea index }(\mathrm{AHI}) \\
\geqslant 34.4 \text { events } \cdot \mathrm{h}^{-1} \text { ) compared with waist-matched } \\
\text { subjects with an } \mathrm{AHI}<34.4 \text { events } \cdot \mathrm{h}^{-1}\end{array}$} \\
\hline & & \multicolumn{2}{|c|}{ AHI events $\cdot h^{-1}$} & \multirow[t]{2}{*}{ p-values } \\
\hline & & $\geqslant 34.4$ & $<34.4$ & \\
\hline \multicolumn{2}{|l|}{ Subjects $\mathbf{n}$} & 40 & 40 & \\
\hline \multicolumn{2}{|c|}{ Age yrs } & $44.4 \pm 8.8$ & $46 \pm 10.2$ & 0.447 \\
\hline \multicolumn{2}{|c|}{ Waist circumference $\mathbf{c m}^{\#}$} & $95.9 \pm 8.7$ & $95.6 \pm 8.6$ & 0.867 \\
\hline \multicolumn{2}{|c|}{$\mathrm{BMI} \mathrm{kg} \cdot \mathrm{m}^{-2}$} & $27.8 \pm 4.0$ & $27.4 \pm 3.4$ & 0.462 \\
\hline \multicolumn{2}{|c|}{ Serum A-FABP levels $\mathrm{ng} \cdot \mathrm{mL}^{-1}$} & $19.2 \pm 8.9$ & $15.9 \pm 4.7$ & $0.036^{\bullet}$ \\
\hline
\end{tabular}

desaturation and minimal oxygen saturation, independent of age and obesity. Increasing serum A-FABP levels were also associated with increased insulin resistance, independent of age and adiposity.

There is increasing evidence in cellular and animal experiments that A-FABP might play a central role in insulin resistance and glucose intolerance $[10,26]$. In animal experiments, the apparent function of A-FABP was mediation of insulin resistance, independent of the effects of obesity and the promotion of atherosclerosis [9, 27]. A-FABP appeared to assume an important position in influencing metabolic responses, and hence lipid and glucose metabolism, and the mediation of subsequent development of atherosclerosis. The present results confirmed that in this cohort of males, the majority of whom had OSA of varying degrees of severity, serum A-FABP level also correlated with waist circumference/ BMI, as previously reported in the general population [18]. The subsequent partial correlation analyses were therefore performed with adjustment for waist circumference and BMI (table 2). The comparisons between groups of different OSA severity, determined according to AHI tertiles, suggest that the difference in serum A-FABP levels mainly existed between those with high AHI of $\geqslant 34.4$ events $\cdot h^{-1}$ and those with AHI $<34.4$ events $\cdot h^{-1}$, while there was no difference between subjects whose OSA would be considered as moderate compared to those with mild/no OSA. There was also significant positive linear correlation between FABP levels and the severity of oxygen desaturation, independent of obesity. These findings suggests that there may be a threshold effect of OSA on FABP levels and that FABP levels may be upregulated mainly in those subjects with high AHI and a more severe degree of hypoxaemia. There is scanty information regarding the regulation of FABP, although there has been previous evidence of FABP expression being regulated by hypoxia in human trophoblasts in vitro [28]. It is possible that the different intensity of intermittent hypoxia in OSA subjects results in different degrees of heightened expression of AFABP, which then assumes a mediating role between OSA and metabolic dysfunction.

The significant correlations between serum A-FABP levels and insulin resistance remained after adjustment for obesity, simulating the finding in animal experiments that serum AFABP deficiency protected against development of insulin resistance, independent of the effects of obesity [14]. An independent association between OSA and insulin resistance has been reported and multiple mechanisms may be involved, in particular intermittent hypoxia, sleep fragmentation, sympathetic overactivity or even activation of the hypothalamic pituitary axis [3, 29, 30]. Multiple biomediators may be involved and A-FABP may well be one of them. As yet, little is known about the regulation of A-FABP. However, the relationships between these pathophysiological mechanisms in OSA and the regulation of A-FABP, and their respective contributions towards the development of insulin resistance, are beyond the scope of the present study's design.

Several strengths and limitations of the current study warrant further discussion. The results of the current study were based on male subjects and should not be generalised to female subjects. Furthermore, only subjects without cardiometabolic 
comorbidities were recruited to avoid the confounding effects of these diseases; this is considered one of the strengths of the present study, given that the major objective was to investigate for any independent relationship between OSA and A-FABP. However, in clinical practice, many OSA subjects will have overt metabolic syndrome or cardiovascular diseases, and the current findings cannot be entirely extrapolated. Another potential limitation is the use of a sleep clinic-based cohort, which may introduce potential sample bias, though this would have the advantage of including a significant number of subjects with a wide range of OSA severity within a not-toolarge sample size. Although the results from the present study were being controlled for age, BMI and waist circumference, it would be more definitive to assess body fat content with more sophisticated and detailed measurements. Given the finding of an independent association between A-FABP levels and severity of OSA in the present study, further definitive information on the cause-effect relationship and interactive pathways may be derived by studying the effects of continuous positive airway pressure treatment of OSA on serum AFABP levels, as well as in vitro studies using cell culture or animal models.

\section{Conclusion}

In summary, the present authors have demonstrated significant differences in serum adipocyte-fatty acid binding protein levels between subjects with severe obstructive sleep apnoea compared to those with no or mild obstructive sleep apnoea in terms of apnoea/hypopnoea index. Serum adipocyte-fatty acid binding protein levels were also found to correlate with nocturnal hypoxaemia and insulin resistance, independent of adiposity. The findings support the hypothesis that adipocytefatty acid binding protein could be a link in the association between obstructive sleep apnoea and cardiometabolic dysfunction.

\section{ACKNOWLEDGEMENTS}

The authors would like to thank A. Lai (Dept of Medicine, University of Hong Kong, Hong Kong) for assistance in statistical analysis and P.P. Ku (Dept of Medicine, University of Hong Kong) for technical assistance in analysis of sleep studies.

\section{REFERENCES}

1 Vgontzas AN, Bixler EO, Chrousos GP. Metabolic disturbances in obesity versus sleep apnoea: the importance of visceral obesity and insulin resistance. J Intern Med 2003; 254: 32-44.

2 Lam JC, Lam B, Lam CL, et al. Obstructive sleep apnea and the metabolic syndrome in community-based Chinese adults in Hong Kong. Respir Med 2006; 100: 980-987.

3 Tasali E, Ip MS. Obstructive sleep apnea and metabolic syndrome: alterations in glucose metabolism and inflammation. Proc Am Thorac Soc 2008; 5: 207-217.

4 Lavie L. Obstructive sleep apnoea syndrome - an oxidative stress disorder. Sleep Med Rev 2003; 7: 35-51.

5 Gozal D, Kheirandish L. Oxidant stress and inflammation in the snoring child: confluent pathways to upper airway pathogenesis and end-organ morbidity. Sleep Med Rev 2006; 10: 83-96.
6 Fantuzzi G. Adipose tissue, adipokines, and inflammation. J Allergy Clin Immunol 2005; 115: 911-919.

7 Boord JB, Fazio S, Linton MF. Cytoplasmic fatty acidbinding proteins: emerging roles in metabolism and atherosclerosis. Curr Opin Lipidol 2002; 13: 141-147.

8 Kazemi MR, McDonald CM, Shigenaga JK, Grunfeld C, Feingold KR. Adipocyte fatty acid-binding protein expression and lipid accumulation are increased during activation of murine macrophages by toll-like receptor agonists. Arterioscler Thromb Vasc Biol 2005; 25: 1220-1224.

9 Uysal KT, Scheja L, Wiesbrock SM, Bonner-Weir S, Hotamisligil GS. Improved glucose and lipid metabolism in genetically obese mice lacking aP2. Endocrinology 2000; 141: 3388-3396.

10 Makowski L, Boord JB, Maeda K, et al. Lack of macrophage fatty-acid-binding protein aP2 protects mice deficient in apolipoprotein E against atherosclerosis. Nat Med 2001; 7: 699-705.

$11 \mathrm{Fu}$ Y, Luo N, Lopes-Virella MF. Oxidized LDL induces the expression of ALBP/aP2 mRNA and protein in human THP-1 macrophages. J Lipid Res 2000; 41: 2017-2023.

12 Hotamisligil GS, Johnson RS, Distel RJ, Ellis R, Papaioannou VE, Spiegelman BM. Uncoupling of obesity from insulin resistance through a targeted mutation in $\mathrm{aP2}$, the adipocyte fatty acid binding protein. Science 1996; 274: 1377-1379.

13 Maeda K, Cao H, Kono K, et al. Adipocyte/macrophage fatty acid binding proteins control integrated metabolic responses in obesity and diabetes. Cell Metab 2005; 1: 107-119.

14 Boord JB, Maeda K, Makowski L, et al. Combined adipocyte-macrophage fatty acid-binding protein deficiency improves metabolism, atherosclerosis, and survival in apolipoprotein E-deficient mice. Circulation 2004; 110: 1492-1498.

15 Cao H, Maeda K, Gorgun CZ, et al. Regulation of metabolic responses by adipocyte/macrophage fatty acid-binding proteins in leptin-deficient mice. Diabetes 2006; 55: 19151922.

16 Furuhashi M, Tuncman G, Gorgun CZ, et al. Treatment of diabetes and atherosclerosis by inhibiting fatty-acid-binding protein aP2. Nature 2007; 447: 959-965.

17 Shum BO, Mackay CR, Gorgun CZ, et al. The adipocyte fatty acid-binding protein $\mathrm{aP} 2$ is required in allergic airway inflammation. J Clin Invest 2006; 116: 2183-2192.

$18 \mathrm{Xu} \mathrm{A}$, Tso AW, Cheung BM, et al. Circulating adipocytefatty acid binding protein levels predict the development of the metabolic syndrome: a 5-year prospective study. Circulation 2007; 115: 1537-1543.

19 Tso AW, Xu A, Sham PC, et al. Serum adipocyte fatty acid binding protein as a new biomarker predicting the development of type 2 diabetes: a 10-year prospective study in a Chinese cohort. Diabetes Care 2007; 30: 2667-2672.

20 Yeung DC, Xu A, Cheung CW, et al. Serum adipocyte fatty acid-binding protein levels were independently associated with carotid atherosclerosis. Arterioscler Thromb Vasc Biol 2007; 27: 1796-1802.

21 Tuncman G, Erbay E, Hom X, et al. A genetic variant at the fatty acid-binding protein aP2 locus reduces the risk for hypertriglyceridemia, type 2 diabetes, and cardiovascular disease. Proc Natl Acad Sci USA 2006; 103: 6970-6975. 
22 Ip MS, Lam B, Ng MM, Lam WK, Tsang KW, Lam KS. Obstructive sleep apnea is independently associated with insulin resistance. Am J Respir Crit Care Med 2002; 165: 670-676.

23 EEG arousals: scoring rules and examples: a preliminary report from the Sleep Disorders Atlas Task Force of the American Sleep Disorders Association. Sleep 1992; 15: 173-184.

24 Sleep-related breathing disorders in adults: recommendations for syndrome definition and measurement techniques in clinical research. The Report of an American Academy of Sleep Medicine Task Force. Sleep 1999; 22: 667-689.

25 Xu A, Wang Y, Xu JY, et al. Adipocyte fatty acid-binding protein is a plasma biomarker closely associated with obesity and metabolic syndrome. Clin Chem 2006; 52: 405-413.

26 Makowski L, Brittingham KC, Reynolds JM, Suttles J, Hotamisligil GS. The fatty acid-binding protein, aP2, coordinates macrophage cholesterol trafficking and inflammatory activity. Macrophage expression of aP2 impacts peroxisome proliferator-activated receptor gamma and IkappaB kinase activities. J Biol Chem 2005; 280: 12888-12895.

27 Boord JB, Maeda K, Makowski L, et al. Adipocyte fatty acidbinding protein, aP2, alters late atherosclerotic lesion formation in severe hypercholesterolemia. Arterioscler Thromb Vasc Biol 2002; 22: 1686-1691.

28 Biron-Shental T, Schaiff WT, Ratajczak CK, Bildirici I, Nelson DM, Sadovsky Y. Hypoxia regulates the expression of fatty acid-binding proteins in primary term human trophoblasts. Am J Obstet Gynecol 2007; 197: 516.

29 Punjabi NM, Polotsky VY. Disorders of glucose metabolism in sleep apnea. J Appl Physiol 2005; 99: 1998-2007.

30 Trakada G, Chrousos G, Pejovic S, Vgontzas A. Sleep apnea and its association with the stress system, inflammation, insulin resistance and visceral obesity. Sleep Med Clin 2007; 2: 251-261. 